\title{
Pentoxyfylline for hepatopulmonary syndrome after liver transplantation: case report
}

\author{
Yi Wei Tan', Rohit Vijay Agrawal', Terry Ling Te Pan${ }^{1}$, Mark Muthiah², Weng Hoa Wong ${ }^{1}$ \\ ${ }^{1}$ Department of Anaesthesia, National University Hospital, Singapore \\ ${ }^{2}$ Department of Gastroenterology, National University Hospital, Singapore
}

Background: Hepatopulmonary syndrome (HPS) is characterized by abnormal arterial oxygenation caused by intrapulmonary vascular dilatations in the setting of liver disease. Even after liver transplantation (LT), recovery from HPS can be protracted. The pathogenesis of HPS is unclear. Several mediators, including tumor necrosis factor- $\alpha$ (TNF- $\alpha$ ), nitric oxide, endothelin-1, and vascular endothelial growth factor, have been implicated. Pentoxifylline is a phosphodiesterase inhibitor with inhibitory effects on TNF- $\alpha$ and NO, and has been linked to improved oxygenation in HPS. Small uncontrolled trials have investigated the effect of pentoxifylline in patients with HPS who had not undergone LT. However, no trials have yet been conducted to investigate the benefit of pentoxifylline in aiding recovery from HPS post-LT.

Methods: We report a case of a 49-year-old man with residual HPS post-LT, who was treated with pentoxyfylline and achieved marked improvement in oxygenation. Our patient was diagnosed with Child's B cirrhosis from chronic hepatitis $\mathrm{C}$ infection at the age of 44. While awaiting transplantation, he was diagnosed with severe HPS. He underwent a deceased donor LT at age 49.

Results: Preoperatively, his $\mathrm{PaO}_{2}$ was $95 \mathrm{mmHg}$ on $4 \mathrm{~L} / \mathrm{min}$ supplemental oxygen via nasal prongs. Postoperatively, his requirements rose significantly-he required $100 \%$ inspired oxygen via a non-rebreather mask to maintain his $\mathrm{SpO}_{2}$ at $92 \%$ and above. Pentoxyfylline was started on postoperative day 6 and continued for 2 weeks. During this period, his oxygen requirements returned to his pre-operative levels, and by 2 months post-LT, he was no longer oxygen-dependent at rest. Pentoxyfylline was well-tolerated by the patient. There were no deleterious effects on the liver graft and no apparent adverse drug interactions. While his improvement in oxygenation could entirely have been a consequence of his $L T$, the sequence of events suggests pentoxyfylline may have had a role to play.

Conclusions: Pentoxyfylline may hasten recovery in patients with residual HPS post-LT.

Corresponding author: Yi Wei Tan

E-mail: tanyiwei@gmail.com

(C) The Korean Society for Transplantation

This is an Open Access article distributed under the terms of the Creative Commons Attribution Non-Commercial License (http://creativecommons.org/licenses/by-nc/4.0/) which permits unrestricted non-commercial use, distribution, and reproduction in any medium, provided the original work is properly cited 